\title{
HUAROCHIRÍ: \\ RECORDANDO LAS VOCES DEL PASADO \\ EN LOS MITOS DE CREACIÓN
}

\author{
POR \\ Nina Gerassi-NavarRo \\ Mount Holyoke College
}

Hábleme. Hay algo maravilloso en su voz.

Hay que volver a la voz.

Oscar Wilde

Las tradiciones orales del pasado tienen el valor de preservar la forma en que una cultura estructura el mundo y reflexiona acerca de sí misma. Transmitidas de generación en generación, ya sea en forma de canción o relato, estos mensajes verbales evocan el pasado, y como tales, se transforman en testimonios de una cultura y de su historia. ${ }^{1}$ En la medida en que estas narraciones orales distinguen entre el pasado y el presente (estableciendo así un orden temporal), y colocan el pasado en directa relación con el presente afirmando y reproduciendo las diferencias entre los hombres y mujeres de la actualidad y aquellos poderosos seres del pasado, dichas tradiciones constituyen la conciencia histórica de una cultura.

Los mitos son aquellas tradiciones vinculadas con el génesis de una comunidad determinada. En estos relatos anónimos se hace presente la genealogía de una determinada comunidad, exponiendo las relaciones entre los diferentes grupos de personas, animales, plantas y espíritus. A través de estos relatos la configuración particular del mundo de esa comunidad queda trazada y su cosmogonía se afirma. Sin embargo, al ser relatos orales, con el paso del tiempo estas narraciones van transformándose. El mito es un modo de conciencia social a través del cual una cultura construye un marco interpretativo y orienta sus actividades sociales. ${ }^{2} \mathrm{Al}$ igual que la historia, el mito asegura que las relaciones de importancia fundamental de una cultura no pasen al olvido. En este sentido, la historia y los mitos son formas complementarias del proceso social interpretativo ya que la conciencia mítica también reflexiona acerca del proceso temporal (pasado versus presente) dentro de un orden social particular. Por ello, el mito surge como metáfora a través de la cual una sociedad se reproduce y dibuja su identidad en el espacio y el tiempo.

\footnotetext{
1 Jan Vansina, Oral Tradition As History (Madison: The University of Wisconsin Press, 1985) $27-$ 29.

${ }^{2}$ Jonathan D. Hill, "Introduction: Myth and History", Rethinking History and Myth: Indigenous South American Perspectives on the Past. Jonathan D. Hill, editor (Urbana: University of Illinois Press, 1988) 1-17.
} 
Evocados a través de la memoria y transmitidos por repetición, estos recuerdos comúnmente se funden con otras narraciones orales, y así se expanden, se desdoblan o inclusive se simplifican, hasta tal punto que muchas veces el relato original se diluye por completo entre los intersticios del proceso de transmisión. Por otra parte, estos cambios son indispensables para presevar el relato oral, pues de otra manera el significado del mito se vuelve oscuro y confuso. ${ }^{3}$ Ésta es la particularidad del discurso oral: "ser un pensamiento en movimiento", 4 en el cual los cambios y enfrentamientos tanto de ideas como de las interacciones humanas recrean la historia de una cultura y definen su identidad. Son voces y ecos que van modulando el proceso de transformación de una cultura.

Rastrear la evolución de una tradición oral de una cultura implica acercarse a esa cultura para desentrañar las distintas influencias que incidieron en su percepción de la realidad y descubrir de qué manera el pasado o los distintos momentos del pasado dejaron su huella en el presente, moldeando la conciencia social de esa comunidad. Es un acercamiento a los procesos cognitivos de una comunidad, entender de qué forma el pasado ha sido reordenado por la conciencia mítica.

Desde la llegada de los españoles a América y la imposición de su palabra escrita como forma de comunicación legítima, las culturas indígenas han estado sometidas a leyes y dictámenes ajenos que exigían una reorganización de su sociedad y cultura siguiendo los parámetros europeos. Sin embargo, casi quinientos años después, numerosas comunidades indígenas mantienen su propia configuración simbólica anclada justamente en su pasado mítico. Estudiar las transformaciones, desde una perspectiva literaria, de algunos mitos de creación que han logrado sobrevivir hasta hoy día, revela de qué forma el encuentro de esas dos culturas, la española y la indígena, impuso un reordenamiento del mundo indígena e inclusive de su historia, pero más importante aún expone la forma en que una comunidad logró mantener su propia voz e identidad a pesar del proceso de aculturación que se les impuso a partir de 1492.

Un valioso testimonio de este proceso de transformación y resistencia se encuentra en la localidad andina de Huarochirí (actual departamento de Lima) sobre la ladera oeste de la cordillera central del Perú. Durante la administración colonial, dicha localidad pertenecía a la provincia de Huarochirí que se extendía desde el río Chillón al norte hasta la provincia de Yauyos al sur, y desde la puna al este hasta el valle de las montañas costeras al oeste. Si bien Huarochirí se hallaba en la ruta principal de Lima hacia el rico centro minero de Potosí, nunca fue parte del centro colonial español y por consiguiente su integración al sistema colonial no fue tan intensa ni abrumadora como la de los grandes centros explotados por los españoles. ${ }^{5}$

\footnotetext{
${ }^{3}$ Goody afirma que para entender un texto antiguo se necesita un aparato de interpretación ya que el lenguaje también cambia. Las culturas orales incorporan este proceso de interpretación en la transmisión de sus relatos de generación en generación. Véase Jack Goody, The Interface Between The Written and The Oral (Cambridge: Cambridge University Press, 1987) 121.

${ }^{4}$ Martín Lienhard, La voz y su huella: escritura y conflicto étnico-social en América Latina (14921988) (La Habana: Casa de las Américas, 1990) 49.

5 Partiendo de las tradiciones narradas en el Manuscrito y de otros documentos, Karen Spalding analiza la conformación de las distintas comunidades de Huarochirí prestando especial atención a la organización social y estructura política de la región: Huarochiri: An Andean Society Under Inca and Spanish Rule, (Stanford: Stanford University Press, 1984) 1-3.
} 
El texto que hoy se conoce como el manuscrito de Huarochirí o "mitos de Huarochiri", es un documento excepcional, no sólo porque es el único texto literario que existe del siglo XVI-XVII escrito en quechua, sino porque además constituye un valioso testimonio del mundo andino prehispánico de Huarochirí. ${ }^{6}$ Desde el origen del mundo y las luchas entre los fundadores, pasando por los distintos rituales y modos de organización social, hasta la llegada de los españoles y el proceso de desplazamiento que ejercieron sus dioses sobre las huacas indígenas, el manuscrito de Huarochirí reconstruye las distintas etapas del pasado, formando un verdadero compendio histórico, religioso, cultural y social de una comunidad andina visto desde su propia interioridad.

Si bien se desconoce con certeza el autor y el proceso escritural del manuscrito, una figura clave relacionada con el texto es la del padre Francisco de Ávila, que fue nombrado vicario y juez eclesiástico de la provincia de Huarochirí en 1597. Ávila llegó con el fin de "instruir" a los indígenas y predicar contra las idolatrías en la provincia. ${ }^{7}$ Mestizo, según lo afirma el virrey Marqués de Montesclaros, y huérfano de padres, es decir, sin linaje que pudiera corroborar su pureza de sangre, Ávila tuvo que apelar a su categoría de expuesto para poder obtener un puesto oficial en la administración colonial. ${ }^{8}$ Es quizás esta necesidad de demostrarse como miembro de la sociedad europea la que lo empuja a dedicarse con tanto esmero a la eliminación de creencias y rituales tradicionales en la provincia de Huarochirí. Durante los primeros años como párroco, su actividad no fue tan virulenta, pero a partir de 1608, luego de numerosos pleitos con la justicia eclesiástica, Avila implementó una dura campaña de extirpación en la cual los curas locales fueron aislados de sus comunidades, se destruyeron sitios e ídolos sagrados y se prohibieron las danzas y canciones de los rituales. ${ }^{9}$

\footnotetext{
${ }^{6}$ Para el presente trabajo las citas procederán de la edición bilingúe del manuscrito, traducida por George Urioste, Hijos de Pariya Qaqa: la tradición oral de Waru Chiri (mitología, ritual y costumbres) 2 tomos, 6 (Syracuse, New York: Foreign and Comparative Studies Program Latin American Series, 1983). Se han consultado además, para la comparación de las distintas versiones, las siguientes ediciones del manuscrito: Frank Salomon and George L. Urioste, The Huarochiri Manuscript: A Testament of Ancient and Colonial Andean Religion (Austin: University of Texas Press, 1991); Gerald Taylor, Rites et traditions de Huarochiri: Manuscrit quechua du début du 17 e siècle (Paris: Editions líHarmattan, 1980).

${ }^{7}$ Para una síntesis de las distintas posiciones con respecto al autor y fecha de composición del manuscrito, véase: Frank Salomon, "Chronicles of the Impossible: Notes on three Peruvian Indigenous Historians", From Oral to Written Expression: Native American Chronicles of the Early Colonial Period, Rolena Adorno, editora (Syracuse, NY: Syracuse University, 1982) 9-39; George L. Urioste, introducción, Hijos de Pariya Qaqa: la tradición oral de Waru Chiri (mitología, ritual $y$ costumbres).

${ }^{8}$ El virrey de Montesclaros escribe "Aunque hay opinión que son conocidos sus padres, él se cuenta por expuesto y en cualquier destos dos caminos es mestizo, según se tiene por más cierto". Citado por Pierre Duviols en su estudio bibliográfico en Francisco de Ávila, Dioses y hombres de Huarochiri: narración quechua recogida por Francisco de Ávila [¿1598?]. Traducción José María Arguedas (Lima, 1966) 218.

9 Ávila fue acusado de exacciones contra los indios, obligándolos a trabajar sin pago y "otras faltas graves en el ejercicio de su cargo". Véase Duviols en Ávila, 220 y también Handbook of South American Indian, Julian H. Steward, editor, The Andean Civilizations, 2 volúmenes (New York: Cooper Square Publishers, 1963).
} 
El rol exacto de Ávila con respeto a la elaboración del texto está aún sin definir. Gran parte del material mítico presente en el manuscrito de Huarochirí se encuentra en su obra titulada Tratado sobre los dioses falsos (1608) en el que Ávila expone la idolatría de Huarochirí para justificar su campaña de extirpación. Por otra parte, Ávila no sólo realizó anotaciones en los márgenes del texto indígena sino que además, su nombre aparece mencionado en el mismo manuscrito. Algunos críticos lo consideran como el autor del texto por mencionar en su Tratado haber recogido personalmente el testimonio de informantes dignos de confianza (Trimborn, Galante). No queda claro, sin embargo, si esos testimonios corresponden específicamente a los del manuscrito. Otros críticos postulan a Ávila como el patrocinador y supervisor del texto (Duviols, Arguedas). Este último clasifica el manuscrito como el resultado de una encuesta realizada por Ávila; otros piensan que fue simplemente el editor (Urioste); también hay quienes creen que Ávila no fue ni patrocinador ni editor, y que el texto quechua fue compuesto por algún indio ladino, bien sea a pedido de Ávila o bien como reacción a su Tratado (Taylor).

Por el momento sólo se pueden ofrecer hipótesis con respecto a este aspecto, pues a pesar de que el manuscrito denuncia aquellos "ídolos" a los que Ávila hace referencia en su Tratado, existen otras voces en el texto que justamente reivindican lo andino, comenzando por el narrador del prefacio, quien parece haber tenido clara conciencia de la importancia que los europeos le otorgaban a la escritura para mantener vivo el pasado: ${ }^{10}$

\begin{abstract}
Si los antepasados de los llamados Indios hubieran tenido cronistas, sus costumbres no se habrían ignorado hasta ahora. Hubiera pasado lo mismo que con los españoles cuyos hechos heroicos son conocidos hasta el presente. Siendo éste el caso, que aún nada se ha escrito hasta hoy, expongo aquí las costumbres de los antepasados de los llamados Waru Chiri, ya que tienen un padre común: qué creencias tenían, cómo viven hasta el presente, y otras cosas por el estilo. Por lo demás, esto se escribirá con relación a cada comunidad en particular, contando cómo viven desde sus orígenes (1).
\end{abstract}

El autor de este pasaje no se identifica explícitamente como miembro de una de estas comunidades, sin embargo muestra un interés concreto en la recuperación y permanencia de las costumbres y vidas de los antepasados de los habitantes de Huarochirí. La introducción afirma que se escribe para que no se pierda ese pasado. Por otra parte, el narrador hace referencia a la diversidad de comunidades que, según él, poseen diferencias tan claras que no se las puede integrar como una unidad sino que deben relatarse sus historias individualmente. Por consiguiente, el manuscrito deberá leerse como el resultado de una recopilación de testimonios orales que evocan las tradiciones de los pobladores Yunca, Checa y Concha que habitaban la región. La diversidad de comunidades, cada una

\footnotetext{
${ }^{10} \mathrm{Si}$ bien la región andina carecía de escritura alfabética, los quipus o nudos de diversos colores y tamaños, sirvieron como formas de documentación, codificando reglas y datos de contabilidad, y se usaron además para recordar hechos históricos. Las investigaciones demuestran que los cantares tradicionales y pinturas también sirvieron para recordar el pasado. Véase María Rostworowski de Diez Canseco, Estructuras andinas del poder. Ideología religiosa y política (Perú: Instituto de Estudios Peruanos, 1983) 101; Regina Harrison, Signs, Songs, and Memory in the Andes. Translating Quechua Language and Culture (Austin: University of Texas Press, 1989) 71-72.
} 
con su historia particular, explicaría la pluralidad de voces, digresiones, contradicciones y diferencias lingüísticas que recorren el texto. ${ }^{11}$ Sin embargo, los recuerdos aparecen ordenados en una secuencia relativamente coherente y cronológica, con referencias intercaladas a pasajes anteriores, otorgándole al texto una determinada unidad.

Es importante tener en cuenta que si bien los mitos son modos de entender los procesos sociales a través de los cuales se crea, se reproduce y se transforma el orden socio-cultural andino, al ser transcritas, como toda tradición oral, sufren una transformación, una especie de traducción y traición, en el que los criterios europeos de orden y unidad tuvieron un efecto determinante. ${ }^{12}$ Por estas razones el aspecto más estudiado del manuscrito ha sido el texto quechua y las distintas posibles traducciones, pues de ellas depende la resolución de cuestiones históricas (fecha y autoría) y el modo en que se interpreta el manuscrito. ${ }^{13}$ Por otra parte, a medida que las investigaciones arqueológicas, lingüísticas y etnográficas avanzan, la lectura del manuscrito ofrece nuevos modos de entender la cultura andina prehispánica que amplían, corrigen y también corroboran la información ofrecida en las crónicas.

De los diversos relatos del manuscrito de Huarochirí, algunos continúan teniendo vigencia hoy día, como lo comprueba la recopilación del investigador Alejandro Ortíz Rescaniere, quien, en 1977, realizó un compendio de los relatos orales de la región de Huarochirí, titulado Huarochiri 400 años después. ${ }^{14}$ Uno de los primeros mitos en ambos

\footnotetext{
$"$ George L. Urioste, Hijos de Pariya Qaqa: la tradición oral de Waru Chiri (mitología, ritual y costumbres). Urioste hace una traducción en la que su propósito es presentar una versión lo más fiel posible al sentido del quechua original. En su introducción, subraya que una de las dificultades del texto es la presencia de palabras del quechua hablado en el Perú central dentro de una estructura gramatical que refleja exclusivamente un dialecto ayacuchano-cuzqueño-boliviano. Frank Salomon, en su ensayo introductorio trata este tema de forma más detallada, especificando los distintos sustratos quechua y no quechua del texto. Traducción Frank Salomon and George L. Urioste, The Huarochiri Manuscript: A Testament of Ancient and Colonial Andean Religion (Austin: University of Texas Press, 1991) especialmente 30-38.

${ }_{12}$ Terence Turner, "Ethno-Ethnohistory: Myth and History in Native South American Representations of Contact with Western Society", Rethinking History and Myth, Jonathan D. Hill, editor (Chicago: University of Illinois, 1988) 235-281.

${ }^{13}$ La primera traducción del manuscrito fue al alemán, iniciada en 1939 y completada en 1941 por Hermann Trimborn, (quien luego, en 1967, presentó una nueva versión con transcripción paleográfica y un estudio sobre los dioses y su culto por Antje Kelm). Un año después, Hipólito Galante tradujo el texto al latín. En 1960, J. Lara incluyó en su Leyendas Quechuas siete relatos del manuscrito, pero la primera traducción completa al español apareció recién en 1966, por José María Arguedas, acompañada por un estudio biográfico sobre el padre Ávila de Pierre Duviols. Siguieron el trabajo de Gérald Taylor al francés, en 1980, y la traducción de Jorge Urioste al español en 1983, versión en la cual me baso para el presente trabajo. A estas traducciones se debe agregar el trabajo reciente de Jorge Urioste y Frank Salomon, quienes en 1991 presentaron una edición bilingüe en quechua e inglés con un nuevo estudio y notas. Para un análisis detallado de las diferencias entre las traducciones véase Roswith Hartmann, "El texto quechua de Huarochirí: una evaluación crítica de las ediciones a disposición", Histórica, 5 (2): 167-208; también Gerald Taylor, "Las ediciones del manuscrito quechua de Huarochirí. Respuesta a Roswith Hartmann”, Histórica, 6 (2): 255-278.

${ }^{14}$ Alejandro Ortiz Rescaniere, Huarochiri, 400 años después (Perú: Pontificia Universidad Católica del Perú, 1980): 22-24.
} 
textos es el mito de creación que relata la historia de dos huacas o figuras sagradas, Cuniraya y Cavillaca. Dicho relato titulado "La vida de Quni Raya el Wira Qucha. Cómo fue Quni Raya Wira Qucha en su tiempo y cómo Qawi Llaqa parió a su hijo y lo que pasó" corresponde al segundo capítulo del Manuscrito. Cuni Raya ya aparece mencionado en el primer capítulo del Manuscrito y se lo identifica con Vira Cocha, "el hacedor" o "creador". Según se informa en el Manuscrito, el episodio entre Cuniraya y Cavillaca transcurre en la antigüedad, "en tiempos muy remotos". Sin embargo, no se sabe cuándo exactamente ubicarlo pues el narrador afirma no saber si Cuniraya vivió antes o después de otra huaca fundadora, Pariacaca. Ilustrando una de las "irregularidades" recurrentes en el texto con respecto a la cronología, posteriormente, en el capítulo 15, se afirma que Cuniraya es el padre de Pariacaca y que fue el creador del mundo ya que "Antes de que él existiera, no había absolutamente nada en este mundo". Es decir que si bien no existe una cronología exacta de la historiade allí la atemporalidad del mito- por otra parte, hay una división temporal entre un pasado, en el que se inicia el origen de un orden jerárquico, y un presente distinto a ese pasado. En este sentido la llegada de Cuniraya, el creador, marca el comienzo de la conciencia mítica de los pobladores de Huarochirí.

Cavillaca, en cambio, parece ser una huaca de menos importancia en la medida en que aparece mencionada de pasada sólo una vez más en los treinta y un capítulos (si se cuentan los suplementos) que componen el manuscrito. Su presencia, sin embargo, es clave ya que su rechazo y posterior huida de Cuniraya dan origen a la conformación del reino animal. Ante la ausencia del objeto deseado, Cuniraya parece llenar ese espacio vacío por medio de un reordenamiento jerárquico del mundo sensible que lo rodea.

La historia es simple: Cavillaca es una "doncella" a quien todos los huacas y wilkas (miembros de la nobleza sacerdotal) desean, pero ella no se deja poseer por nadie. Un día, mientras Cavillaca estaba sentada tejiendo bajo el lúcumo, Cuniraya, apodado "el astuto", se convierte en pájaro y pone su semen en el fruto del lúcumo. La lúcuma cae, Cavillaca la come y así queda fecundada sin contacto ninguno. $\mathrm{Al}$ año, Cavillaca reune a todos las huacas de la zona para averiguar quién es el padre de su hijo. ${ }^{15} \mathrm{La}$ importancia de averiguarlo se debe a que es la línea paterna la que establece la descendencia del linaje. El parentesco a su vez es lo que define la pertenencia a un ayllu en particular, considerada la unidad política y productiva básica en la sociedad andina. ${ }^{16}$ Entre las huacas que estaban sentados muy "engalanados" se hallaba también Cuniraya, disfrazado de pobre. Como nadie responde a la pregunta de Cavillaca “¿fuiste tú quien engendró a mi hijo?” decide que sea el mismo niño quien reconozca a su padre. Al dejarlo, éste va gateando por entre las huacas y al llegar a Cuniraya, se alegra de inmediato y se le sube a las faldas. Cavillaca, horrorizada de que ese "pobretón" haya sido su progenitor, toma al niño en brazos y sale corriendo hacia el mar. Cuniraya sale tras ellos vestido ahora con sus túnicas relucientes. El encuentro no se lleva a cabo porque al llegar a la costa, en el lugar marcado por dos rocas, Cavillaca y su hijo quedaron convertidos en piedra. Sin embargo, la historia continua enfocándose en la trayectoria de Cuniraya. En el camino éste se encuentra con distintos animales y les

\footnotetext{
${ }^{15} \mathrm{Si}$ bien todas las traducciones transcriben "hijo", Urioste aclara que el texto no especifica el sexo del hijo de Cavillaca porque la palabra quechua "churi" significa hijo o hija del padre.

${ }^{16}$ Ziudema 22; Spalding 27-30.
} 
pregunta a cada uno si han visto a Cavillaca. Según la respuesta que le ofrecen, o sea si se encuentra cerca o lejos de ella, Cuniraya bendice o maldice a los animales. De esta forma se establece la división del reino animal, es decir que se establece un orden jerárquico en el mundo sensible.

Si bien en el texto no hay una división explícita, aquí termina lo que podría llamarse la primera parte del mito que coincide, aunque de forma más sintética, con la versión contemporánea documentada por Ortíz Rescaniere. A partir de este momento, el ritmo del relato se acelera y las acciones de Cuniraya se enumeran con más rapidez. Cuniraya llega a la costa y se acuesta con la hija de Urpay Wachaq, una huaca que había ido a visitar a Cavillaca en la profundidad del mar. Frustrado por no poder llegar a Cavillaca como Urpay Wachaq, Cuniraya tira todos los peces que la huaca criaba en su casa y desde entonces, afirma el narrador, se encuentra toda clase de peces en el mar. Urpay Wachaq decide vengarse y con la intención de tirarlo en un abismo, atrae a Cuniraya diciéndole que lo va a despiojar. ${ }^{17}$ Pero Cuniraya, "el astuto", se da cuenta de la treta y se salva. La historia termina haciendo referencia a la actividad de Cuniraya quien siguió engañando a muchos pueblos y personas por mucho tiempo.

El "mito de Cuniraya" ejemplifica el modo de construcción del relato oral. Una de las características principales de las culturas orales es estructurar las historias siguiendo determinados patrones que facilitan la memorización, de allí la abundancia de repeticiones, antítesis, paralelismos, epítetos y frases expresivas. ${ }^{18}$ Lo que no se repite o lo que no conlleva a una percepción visual termina por perderse. A ello hay que agregar las formas particulares en que cada cultura concibe y elige organizar su mundo en formas tangibles además de los cambios que, con el tiempo, se van incorporando al relato.

Según los investigadores, las estructuras binarias y triádicas son formas claves de clasificación para la cultura andina. ${ }^{19}$ La oposición hanan/hurin, arriba/abajo, norte/sur, representa simultáneamente lo masculino o superior por un lado, y lo femenino o inferior por otro, y constituye lo que Tom Ziudema llama la estructura básica de la cultura incaica. Otra clasificación binaria importante es la de saya/suyu, hacia arriba y hacia abajo, que define la oposición dentro-fuera. Según Ziudema, las clasificaciones triádicas se obtienen por una serie de términos que pueden ser usados como sinónimos. El resultado de la combinación de las estructuras binarias entre sí o con estructuras triádicas crea una multiplicidad sumamente compleja de divisiones, de las cuales el modelo cuaternario y el séxtuplo son los más característicos.

Teniendo en cuenta esta forma clasificatoria, las parejas opuestas y complementarias que estructuran el mito antiguo de Cuniraya resultan evidentes. En primer lugar, la pareja Cuniraya y Cavillaca representa la oposición hombre-mujer, sol-tierra. Cada uno se destaca de los demás seres que lo rodean. Cavillaca, por su belleza y por negarse a todos sus

\footnotetext{
${ }^{17}$ Para un análisis revelador de la simbología del piojo véase Marie-France Souffez, "El simbolismo del piojo en el mundo andino. Boceto Filológico", Anthropologica 3 (1985): 171-202 y "El piojo y la conversación", Anthropologica 6 (1988): 43-65.

${ }^{18}$ Walter Ong, Orality and Literacy: The Technologizing of the Word (London: Routledge, 1989) 34.

${ }^{19}$ R. T. Ziudema, "Mito e historia en el antiguo Perú", Allpanchis 10 (1977): 15-52.
} 
pretendientes, y Cuniraya, al ser una deidad itinerante que viajaba por el mundo en forma de "hombre muy miserable", se opone a las otras huacas que son deidades locales. A la vez, estas huacas, que parecen ser ricas por la vestimenta que llevan, terminan sin nada, mientras que Cuniraya, a pesar de su traje harapiento, es en realidad rico y es quien consigue poseer a Cavillaca. La oposición entonces se presenta en términos de lo que no se ve y de lo que hay, entre el parecer y el ser. La intervención del hijo entre la pareja divina establece una tríada y evoca por otra parte, el tema del "encuentro" de dioses contrapuestos y las consecuencias que esto acarrea: el ineludible desplazamiento de una de las divinidades (lo que puede leerse como el pasaje de un estado a otro) y la creación del mundo sensible. Por otra parte, el mito también puede representar el recorrido de las aguas desde las sierras hasta las fértiles tierras bajas de los yungas ya que ese es el camino que sigue Cuniraya.

La clasificación por oposiciones no sólo ayuda a visualizar el relato en términos de espacios definidos, aquí/allá, arriba/abajo, refleja además, un aspecto clave de la conceptualización de la identidad andina en la medida en que la identidad cultural, social y política de una comunidad estaba íntimamente vinculada a un espacio geográfico definido. La determinación del parentesco del hijo de Cavillaca es casi un elemento secundario. Lo que el relato parece subrayar más explícitamente es que en el proceso de establecer el linaje y pertenencia, se delimitan los confines territoriales que son los que a su vez definen en gran parte la identidad de un grupo social determinado.

La configuración del reino animal ejemplifica la combinación séxtuple. Seis son los animales que Cuniraya encuentra en el camino. El primero es el cóndor que, al informar a Cuniraya que está cerca de Cavillaca, recibe una alabanza "Tu vivirás para siempre. Solamente tú te comerás a todos los animales de puna cuando mueran, gauanacos y vicuñas y otros animales semejantes ... [y] si alguien alguna vez te matara, ése también morirá". Sigue la zorrina, la que Cuniraya maldice asegurándole que andará sola de noche y apestando. El texto continúa alternativamente asignándole al puma cualidades positivas y al zorro, negativas; al halcón, positivas y a los papagayos, negativas. La creación de los peces no parece responder al mismo tipo de estructura que las anteriores, quizás porque si bien Cuniraya era una deidad identificada con el agua, otro mito antiguo ubica a Urpay Wachaq, la diosa costeña, como la madre de los peces y aves marinas. En este caso se podría pensar en una superposición de cultos a través del tiempo, lo cual era muy común en la cultura andina. ${ }^{20}$

En este punto comienzan las discrepancias entre el mito original y la versión contemporánea documentada por Ortiz Rescaniere. Relatada por el informante Héctor Chuquimuni de aproximadamente 60 años de edad, la historia de Cuniraya, coincide con relativa similitud con la primera parte del mito original, eliminando por completo la referencia a Urpay Wuachac, madre de los peces y aves marinas. Curiosamente, la historia de Cuniraya y Urpay Wuachac aparece referida en otro mito contemporáneo independiente.

La discrepancia fundamental entre ambos relatos de la historia de Cuniraya consiste en la simplificación de la estructura del mito original y la sustitución de referencias específicamente locales por otras de procedencia europea, en particular aquellas referentes a la Biblia. Esto resulta evidente en la escena de la concepción, pues en lugar de que

${ }^{20}$ Rostworowski, 87. 
Cavillaca sea fecundada por el fruto del lúcumo, en la versión contemporánea, Cuniraya le regala una manzana. ${ }^{21}$ No sólo se elimina la voz andina ("lúcuma" es una palabra quechua), sino que además se excluye la referencia al trabajo de Cavillaca, una de las actividades andinas más importantes: la del tejido. La ofrenda de tejidos a los dioses, durante los sacrificios y muchos de los ritos y ceremonias, refleja la importancia de esta actividad en el ámbito andino. Murra señala que "de todas las etapas vitales, la muerte tenía la más íntima relación con el tejido". ${ }^{22}$ De hecho, una de las cualidades asignadas a Cuniraya Viracocha es la de presidir y protejer a los artesanos dedicados al telar. Por otra parte era costumbre de los dioses de la mitología andina recorrer pueblos y regiones vestidos en harapos, y en el caso de Viracocha se ha relacionado la palabra "cocha" con la raíz aymara kochallo que significa "handrajo, trapo, vestido muy roto". ${ }^{23}$ El hecho de que Cavillaca esté tejiendo en el momento de su fecundación podría verse como la unión de las dos fases fundamentales del relato: el nacimiento y la muerte. Es el pasaje de un estado a otro, ya que la muerte simbólica de Cavillaca, convertirse en piedra, representa la transformación de su espíritu y el comienzo de una nueva etapa.

Otro aspecto del mito que se ha reducido es la conformación del mundo animal. La estructura séxtuple se halla reducida a la mitad, quedando sólo tres animales: el zorro, que mantiene sus cualidades negativas, y el cóndor y el león con connotaciones positivas. El puma, uno de los animales más ricos en cuanto a su simbología en la cultura andina, es sustituido, en la versión moderna, por la figura de máxima realeza europea, el león. ${ }^{24}$ Asimismo, las connotaciones de cada animal están dadas en términos de jerarquía absoluta: el león será el "jefe de los animales de la tierra" y el cóndor, el "rey de las aves”, mientras que el zorro será un animal perseguido por el hombre y de mal agüero.

La clasificación del reino animal en categorías polares extremas refleja el proceso de aculturación al que ha sido sometido el mundo indígena. El pensamiento andino, a diferencia del cristiano, no estructura el mundo en términos absolutos, lo cual no implica que no haya existido una estructura jerárquica. Pero una de las características esenciales de la religiosidad andina es la multiplicidad de divinidades además de una jerarquía de divinidades subalternas, propias de los ayllus y de cada pueblo. De allí que existieran dioses o huacas superpuestos con funciones y connotaciones distintas, dependiendo de la comunidad y de la región. Este hecho dificultó en extremo el proceso de evangelización de los españoles, particularmente en lo que se refería al origen de los distintos pueblos. Al no existir la idea abstracta de Dios en la cultura andina ni vocablo que lo expresara, los españoles optaron por recurrir a una de las divinidades incaicas mayores, Viracocha, para hacer inteligible la

\footnotetext{
${ }^{21}$ El hecho de que en ambos casos, Cavillaca es fecundada sin contacto físico, es uno de los índices que revela la influencia cristiana en el momento en que se inicia la escritura del manuscrito.

${ }_{22}$ John Murra, "La función del tejido en varios contextos sociales y políticos", Formaciones económicas y políticas del mundo andino (Perú: Instituto de Estudios Peruanos, 1975) 152.

${ }^{23}$ Rostworowski, 36.

${ }^{24}$ Tom Ziudema, "The Lion in the City: Royal Symbols of Transition in Cuzco", Gary Urton editor, Animal Myths and Metaphors in South America (Salt Lake City: University of Utah Press, 1985) 145-182; Gary Urton, "Animal Metaphors and the Life Cycle in an Andean Community", Animal Myths, 251-284.
} 
idea de Dios, Padre Todopoderoso que ellos querían imponer. El resultado fue una deformación de la cosmovisión andina. ${ }^{25}$

El recorrido de Cuniraya, del este al oeste y de arriba hacia abajo, es el mismo en ambas versiones, pero en el relato contemporáneo las indicaciones son mucho más específicas. En el mito original, cuando Cavillaca se dirige hacia el mar desde la sierra, Cuniraya simplemente la sigue, mientras que en la versión moderna el recorrido de Cuniraya es más exacto: “A cuatro leguas de distancia pasando por Santo Domingo de los Olleros, rumbo a Chaumari ... atrás ... cuatro leguas, iba siguiéndola el indio Cuniraya". La necesidad de especificar el camino y de definir a Cuniraya como "huarochirano" podría verse como una forma de re-apropiar el territorio propio frente al desplazamiento espacial impuesto por la organización política y social de los españoles.

Otra diferencia entre las dos versiones es la relación posicional de Cuniraya y Cavillaca. En el primer relato la oposición es entre huacas "hermanos" uno, pobre y despreciado y la otra, hermosa y deseada. Como constata Rostworowski al analizar la mitología andina, las parejas divinas no formaban parejas conyugales sino que persistía el rasgo general de hermano/hermana y de madre/hijo. ${ }^{26}$ En cambio, en la versión documentada por Ortiz Rescaniere, Cuniraya es un indio y Cavillaca, una campesina. "La ñusta Cavillaca era de por aquí, de Anchicocha. Cavillaca era hermosa y no le iba a hacer caso al indio de Cuniraya que era feo y andaba haraposo. Por eso Cuniraya le regaló una fruta, una manzana". En esta versión Cuniraya ya no recorre el mundo disfrazado de pobre para engañar a la gente, simplemente "es feo"; y en lugar de usar su astucia para conseguir a Cavillaca, debe recurrir a la manzana casi como forma de compensar su fealdad y ser indio. El juego entre apariencia y realidad de la primera versión (Cuniraya parece pobre pero tiene poderes) se ha desvanecido. Pero quizás más importante aún es que la historia ya no se eleva a un plano sagrado, refiriéndose a dos huacas, sino que reposiciona lo indígena asignándole el plano social definido: el del campesino. Del mismo modo, en lugar de que Cavillaca reúna a todos las huacas de la zona, en la versión moderna reúne a los curacas o jefes locales. Esta sustitución es sumamente significativa, si se tiene en cuenta que fueron los curacas quienes, durante la colonización, ocuparon el rol de mediadores entre las autoridades españolas y los indígenas asegurando que los tributos y otras demandas políticas y económicas españolas se cumplieran. ${ }^{27}$

Si bien la jerarquía política y socio-cultural de los españoles ha desplazado la referencia sagrada de lo indígena, el mito queda contenido dentro de los parámetros establecidos por

\footnotetext{
${ }^{25}$ Pierre Duviols, "Los nombres quechua de Viracocha, supuesto 'Dios Creador' de los evangelizadores”, Allpanchis 10 (1977): 53-63; Harrison, 94-95; Sabine MacCormack, Religion in the Andes. Vision and Imagination in Early Colonial Peru (Princeton: Princeton University Press, 1991) 108-109.

${ }^{26}$ Rostworowski, 15.

${ }^{27}$ Franklin Pease, El Pensamiento mítico (Lima: Mosca Azul, 1982) 19-23; Rostworowski, 99-106; Irene Silverblatt, "Political Memories and Colonizing Symbols: Santiago and the Mountain Gods of Colonial Peru", Hill 174-194. Para un análisis sobre el rol de los curacas durante la colonización, véase también Karen Spalding, "Social Climbers: Changing Patterns of Mobility among Indians of Colonial Peru", Hispanic American Historical Review 50: 645-54.
} 
lo propiamente indígena. Si lo español ha impuesto la reducción de las divinidades andinas a un sólo dios, la pareja divina de Cuniraya y Cavillaca simplemente ha tenido que trasladarse al espacio que los españoles les asignaron a los indígenas: el del indio y el campesino. La relación entre Cuniraya y Cavillaca en el mito original formulada en términos del ser y el parecer (o sea, marcada por una "aparente" desigualdad), sigue vigente en la versión contemporánea pero responde a la clasificación jerárquica española colonial. El indio (Cuniraya disfrazado) representa la posición más baja de la escala social, mientras la categoría de campesina (la ñusta Cavillaca) implica una incorporación y por lo tanto una aceptación dentro del mismo sistema productivo impuesto por los españoles.

Las dos versiones mantienen un claro paralelismo en la medida en que Cuniraya, a pesar de ser indio, es quien le asigna a cada animal su característica particular y es quien establece un orden jerárquico del mundo sensible a través de la palabra. Y en ambas narraciones Cavillaca termina transformándose en huaca al convertirse en piedra. En conclusión, si bien la organización simbólica y los cambios del orden jerárquico que se definen dentro de la cultura indígena no permanecen ajenos al esquema impuesto por los españoles, están explicados dentro de sus propios términos. El resultado del reordenamiento está claramente influído por el contacto con la cultura-otra, pero no el modo en que ese reordenamiento se lleva a cabo. Ese proceso de transformación permanece dentro del espacio y conceptualización simbólica de la cultura indígena.

Ambos relatos revelan que las estructuras de los sistemas socio-culturales andinos surgen como consecuencia de procesos históricos y sociales que involucran conflictos y cambios. Al comparar una versión con otra, las modificaciones que se perciben en la versión contemporánea revelan el efecto que ejerció la cultura española sobre la indígena; del mismo modo en que el encuentro entre divinidades opuestas produjo un nuevo orden, el contacto entre las culturas prehispánicas y sociedades europeas produjo una nueva estructura social. Pero más que la influencia de una cultura sobre otra, la comparación de estas dos versiones del mito de Cuniraya y Cavillaca revela el modo en que las culturas andinas han entendido el encuentro con la cultura española y han sabido cambiar y modificarse para incluir lo propio en el nuevo orden impuesto. La comparación de los mitos de Huarochirí a través de las articulaciones internas del relato oral y el contexto histórico en el cual ambas versiones se registraron, ejemplifica el modo en que la cultura andina ha logrado retener su propia voz y tradición. Poder recuperar hoy, casi cuatrocientos años después, los mitos de un pasado fundacional revela la vigencia y fuerza de la palabra. Es el poder de la voz, sin el confinamiento de la escritura, la que se dibuja una y otra vez por el espacio, entretejiendo pasado y presente y evocando los murmullos y silencios de aquellas culturas que continúan proyectándose a través de las huellas de sus antepasados. 
